\title{
Isosceles Planar Subsets
}

\author{
P. Fishburn
}

AT\&T Labs-Research, Florham Park, NJ 07932, USA

fish@research.att.com

Communicated by János Pach

\begin{abstract}
A finite planar set is $k$-isosceles for $k \geq 3$ if every $k$-point subset of the set contains a point equidistant from two others. There are three nonsimilar 3-isosceles sets with five points and one with six points. Eleven 4-isosceles sets with eight points are noted, and it is conjectured that no 4-isosceles set has nine points. Exactly one 4-isosceles 8-set has four points on a line, and every 4-isosceles set that includes the vertices of a square has fewer than eight points.
\end{abstract}

\section{Introduction}

For $k \geq 3$, a set in $\mathbb{R}^{2}$ is $k$-isosceles if every $k$-point subset includes a 3 -set in which one point is equidistant from the other two. We consider cardinally maximum planar sets that are $k$-isosceles for small $k$. Restrictions on the set such as linearity or convexity will be noted later. The note is motivated by the following conjecture:

Conjecture 1. No 9-point planar set is 4-isosceles.

Although no 4-isosceles 9-set is known, there are at least 11 nonsimilar 4-isosceles planar sets with eight points: see Fig. 1. Conjecture 1 arose from conversations with Paul Erdös on extremal problems in combinatorial geometry, one of his favorite topics [2]-[7]. The Erdös problem that resulted in the conjecture is bracketed by two others in the following trio:

Problem 1. Specify the maximum 3-isosceles planar sets. 

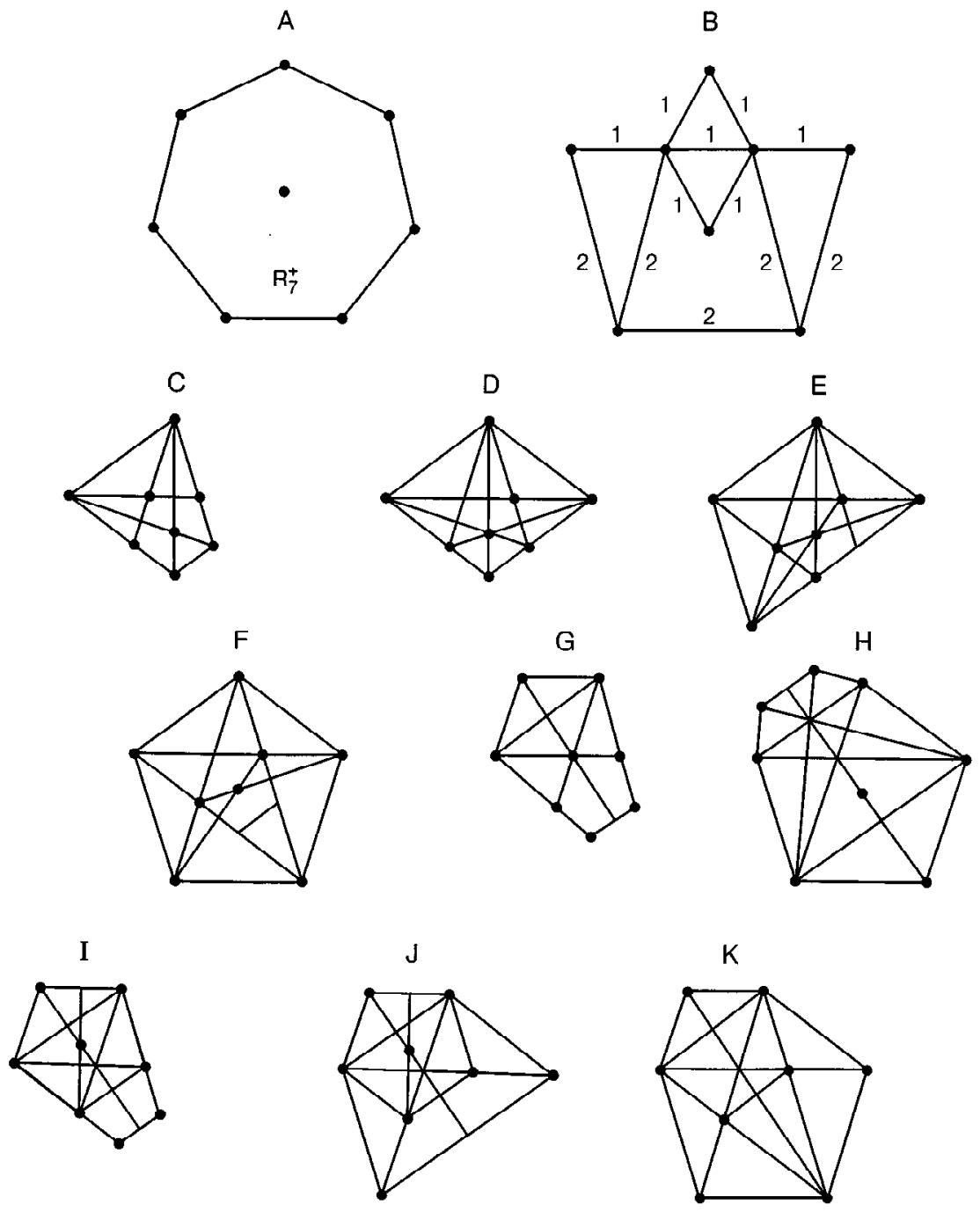

Fig. 1. Eleven 4-isosceles 8-point sets.

Problem 2. Specify the maximum 4-isosceles planar sets.

Problem 3. Specify the maximum planar sets which have a duplicated distance in every 4-set.

Let $d(x, y)$ denote the Euclidean distance between $x$ and $y$ in $\mathbb{R}^{2}$. The equality $d(x, y)=d(z, w)$ for 4-set $\{x, y, z, w\}$ satisfies the duplicated-distance condition of Problem 3 but has no bearing on Problem 2. The vertices of a nonsquare rectangle form the simplest 4-set that has a duplicated distance but is not 4 -isosceles. 
Let $R_{n}$ denote the vertex set of a regular convex $n$-gon, and let $R_{n}^{+}$be $R_{n}$ plus its center point. The unique solution to Problem 1 [6] is $R_{5}^{+}$. There are at least three 12-point sets, including $R_{11}^{+}$, in which every 4 -set has a duplicated distance, and we conjecture [6] that 12 is the maximum for Problem 3.

Aspects of Problem 2 are discussed in the next three sections. The first considers planar sets with four or more points on a line and shows that Fig. 1(B) is the only 8-point 4-isosceles set of this kind. The second is motivated by the fact that $R_{7}^{+}$and Fig. $1(\mathrm{H})$ have no three points on a line. The third section, which reflects the observation that no set on Fig. 1 includes a square, first identifies the three 3 -isosceles sets with five points, one of which is $R_{4}^{+}$. It then argues that a 4 -isosceles set that includes $R_{4}$ or $R_{4}^{+}$has at most seven points. A brief list of open problems concludes the paper.

Because of space constraints and some case-intensive proofs, it will be necessary to omit some proof details.

\section{Linear Restrictions}

The following theorem explains why Fig. 1 has only one set with four points on a line.

Theorem 1. No set of six points on a line is 4-isosceles. A 5-set on a line is 4-isosceles if and only if its adjacent interpoint distances are similar to 2, 1, 1, 2 or 1, 1, 1, 3 or 1, 1, 2, 3. The maximum 4-isosceles sets with five points on a line have seven points. There are two such 7-sets: both have distance pattern 2, 1, 1, 2 on the main line segment, with the other two points both distance 1 or distance 3 from that segment on its perpendicular bisector. There is a unique maximum 4-isosceles set with four points on a line: see Fig. 1(B).

Proof. Denote $n$ points left to right on a horizontal line by $1,2,3, \ldots$ with minimum interpoint distance 1 . It is elementary to show that $\{1,2,3,4,5\}$ is 4-isosceles if and only if its successive interpoint distances are the first four terms in one of $21123,1113 *, 31113$, $1123^{*}$, and 32112. When $n=6$, we can assume that the first four terms apply to $\{1, \ldots, 5\}$. The fifth term, if not $*$, shows the only one-term continuation for which $\{2,3,4,5,6\}$ is 4 -isosceles. In each of the three cases, $\{1,2,3,6\}$ is not 4-isosceles, so no 6-set on a line is 4 -isosceles.

Suppose $n=5$ and the interpoint distance pattern is 2112. Let $p$ denote a sixth point above the line containing 1 through 5 . If $p$ is left of $\perp_{15}$, the perpendicular bisector of $[1,5]$, consideration of $\{p, 3,4,5\}$, and then $\{p, 1,2,5\},\{p, 1,4,5\}$, and $\{p, 1,3,4\}$, shows that $\{1, \ldots, 5\} \cup\{p\}$ is not 4 -isosceles. On $\perp_{15},\{p, 1,2,3\}$ and $\{p, 1,3,4\}$ show that $\{1, \ldots, 5\} \cup\{p\}$ is 4-isosceles if and only if $d(p, 3)=1$ or $d(p, 3)=3$, and only the two distance-1 or two distance-3 points yield a 4-isosceles 7-set. Similar analyses show that the linear successive distance patterns 1113 and 1123 have no off-line $p$ that is 4 -isosceles along with $\{1, \ldots, 5\}$.

Suppose $n=4$. We assume without loss of generality that the adjacent distances between points are $d(1,2)=d(2,3)=1$ and $d(3,4)=\lambda \geq 1$. The only patterns that might have four or more off-line points that are 4-isosceles along with $\{1,2,3,4\}$ occur when $\lambda \in\{1,2\}$. When $\lambda=2$, there is one 7-point 4 -isosceles set, but a detailed 


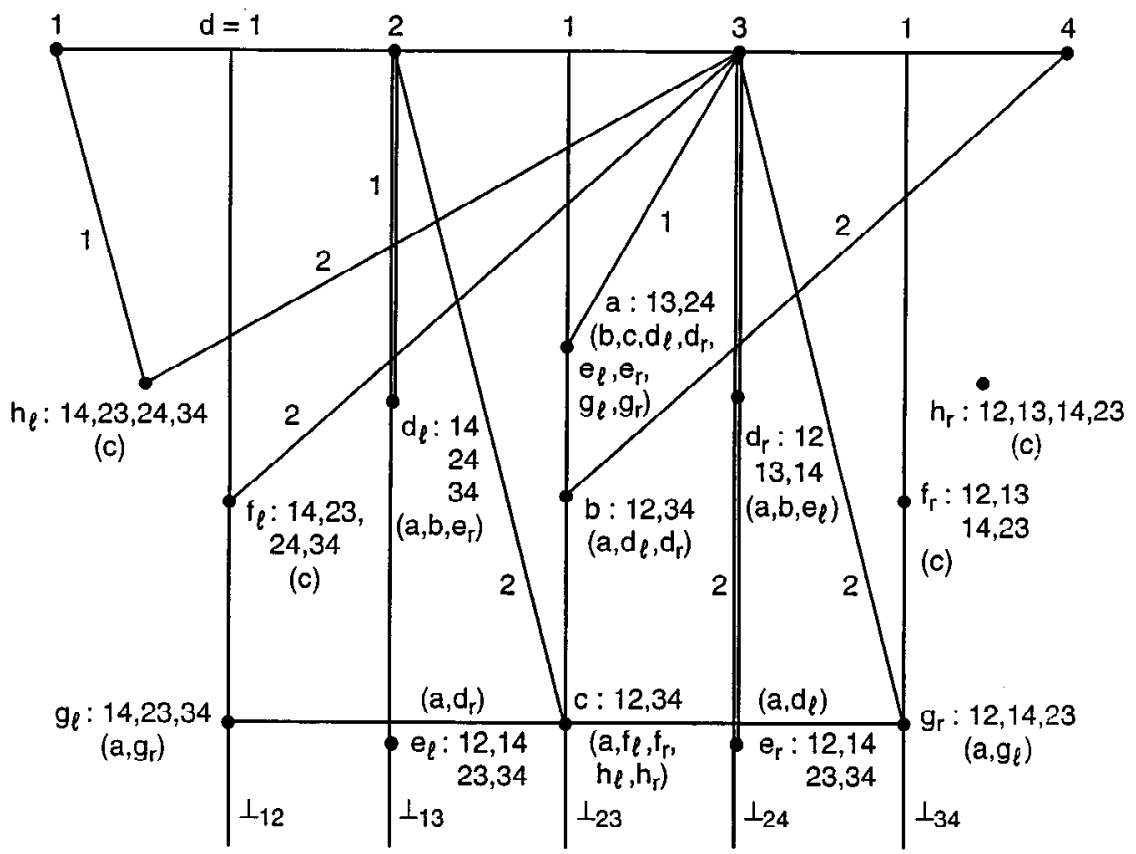

Fig. 2. Analysis for four evenly spaced collinear points.

check on possibilities shows that there is no 8-point 4-isosceles set. The 7-point set has $\{1,2,3,4\}$, the two points on $\perp_{13}$ distance 1 from point 2 , and one point on $\perp_{14}$ distance 1 from point 3 . Suppose $\lambda=1$. An analysis of points below [1, 4] shows that only those marked on Fig. 2 need consideration: we have $a, b, c$ and 10 others symmetric about $\perp_{23}$. Each point is listed with the pairs in $\{1,2,3,4\}$ for which it is not isosceles. Parentheses identify the other points below [1,4] such that the focal point, a point in its parentheses, and $\{1,2,3,4\}$ form a 4 -isosceles 6 -set. To arrive at this point for $\lambda=1$, we consider all points below $[1,4]$ that are 4 -isosceles in conjunction with $\{1,2,3,4\}$ and eliminate those that cannot be part of a 4-isosceles 6-set along with $\{1,2,3,4\}$ and another point below $[1,4]$. Up to symmetry around $\perp_{23}$, there are three 3 -sets below $[1,4]$ each point in which has the other two in parentheses: $\left\{a, g_{\ell}, g_{r}\right\},\left\{a, d_{\ell}, e_{r}\right\}$, and $\left\{a, b, d_{\ell}\right\}$. The last of these has $\left\{1, a, b, d_{\ell}\right\}$ as a nonisosceles 4-set, but the first two yield 4-isosceles 7-sets in conjunction with $\{1,2,3,4\}$.

Let $x^{*}$ denote the mirror image above $[1,4]$ of point $x$ below $[1,4]$ around the line through 1 and 4. Analysis of $\left\{a, d_{\ell}, e_{r}\right\} \cup\{1,2,3,4\}$ shows that no point above $[1,4]$ forms a 4 -isosceles 8 -set with those seven. Moreover, $a^{*}$ is the only point above $[1,4]$ that does this with $\left\{a, g_{\ell}, g_{r}\right\} \cup\{1,2,3,4\}$ : see Fig. 1(B). Hence there is a unique 4-isosceles 8 -set for $n=4$ that uses three points on one side of [1,4] and one point on the opposite side.

To check for 4-isosceles 8-sets with two points above and two below [1, 4], we consider pairs like $\left\{h_{\ell}, c\right\}$ and $\left\{g_{\ell}, g_{r}\right\}$ below [1,4] that give 6-point 4-isosceles sets in 
conjunction with $\{1,2,3,4\}$. We begin with $\left\{h_{\ell}, c\right\}$. The only points above $[1,4]$ that might preserve 4-isoscelesness when added to $\left\{1,2,3,4, h_{\ell}, c\right\}$ are $c^{*}, h_{\ell}^{*}$, and $h_{r}^{*}$, but neither $\left\{h_{r}^{*}, h_{\ell}, 1,4\right\}$ nor $\left\{c^{*}, h_{\ell}^{*}, c, h_{\ell}\right\}$ is 4-isosceles. Similar analyses of $\left\{f_{\ell}, c\right\},\left\{d_{\ell}, a\right\}$, $\left\{d_{\ell}, b\right\},\left\{g_{\ell}, a\right\},\left\{e_{\ell}, a\right\},\{a, b\}$, and $\{a, c\}$ show that none of these can be paired with two points above [1,4] to produce an 8-point 4-isosceles set. This eliminates $a, b, c$ and $a^{*}, b^{*}, c^{*}$ from further consideration, leaving only pairs similar to $\left\{g_{\ell}, g_{r}\right\}$ and $\left\{d_{\ell}, e_{r}\right\}$. Since none of $\left\{g_{\ell}, g_{r}, g_{\ell}^{*}, g_{r}^{*}\right\},\left\{g_{\ell}, g_{r}, d_{\ell}^{*}, e_{r}^{*}\right\},\left\{d_{\ell}, e_{r}, d_{\ell}^{*}, e_{r}^{*}\right\}$, and $\left\{d_{\ell}, e_{r}, d_{r}^{*}, e_{\ell}^{*}\right\}$ is 4-isosceles, no above pair and below pair form a 4-isosceles set along with $\{1,2,3,4\}$.

\section{Circles, Lines, and Convex Polygons}

A finite subset of $\mathbb{R}^{2}$ is convex if its points are the vertices of a convex polygon. We know of no convex 4-isosceles 8-set, but Figs. 1(A) and 1(H) show that there are convex 4-isosceles 7-sets. All such 7-sets besides $R_{7}$ determine at least five distances because $R_{7}$ is the only convex 7-set that determines three distances [1] and none of the five convex 7 -sets that determine four distances ( $R_{8}$ minus a point; versions of $R_{9}$ minus two points) [7] is 4-isosceles. The convex heptagon of Fig. 1(H) has six distances. Other convex 4-isosceles 7-sets are noted in the next section.

Erdös often asked questions about sets with no four points on a circle. The two 7-sets in Theorem 1 for five points on a line show that there are 4-isosceles sets with six points, no four on a circle. Are there any with seven points? Examination of Fig. 1 shows that whenever any two points are removed from an 8-set on the figure, the other six have four on a circle, so the largest subset of Fig. 1 without four points on a circle has only five points.

Set $\left\{2,3,4, a, d_{\ell}, e_{r}\right\}$ of Fig. 2 is a 4-isosceles 6-set with no four points on a circle and no four points on a line. I have no example of a 4-isosceles 6-set with no four points on a circle and no three on a line.

\section{Three-Isosceles Sets and the Square}

The only 3-isosceles 6-set is $R_{5}^{+}$.

Theorem 2. The only 3-isosceles 5-sets are $R_{4}^{+}, R_{5}$, and $R_{5}^{+}$minus a vertex.

Proof. Let $X=\{1,2,3,4,5\}$. Assume that $X$ is 3-isosceles. Denote by $m$ the maximum number of points in $X$ equidistant from another point in $X$. Then $m \in\{2,3,4\}$. If $m=4$, $X$ is easily seen to be $R_{4}^{+}$or $R_{5}^{+}$minus a vertex.

Suppose $m=2$. Because $X$ has 10 triples, for each $i \in X$ there is a partition $\{\{x, y\},\{z, w\}\}$ of $X \backslash\{i\}$ with $d(i, x)=d(i, y) \neq d(i, z)=d(i, w)$. Beginning from $d(1,2)=d(1,3)=a$ and $d(1,4)=d(1,5)=b, a \neq b$, it is straightforward to show that $X$ determines only distances $a$ and $b$. Theorem 1 in [4] says that $X=R_{5}$.

Suppose $m=3$. We assume $d(1,2)=d(1,3)=d(1,4)=a$ and $d(1,5)=b, a \neq b$. Then, for each $i \in\{2,3,4\}$, either $d(i, 5)=d(i, 1)=a$ or $d(i, 5)=d(1,5)=b$. The 


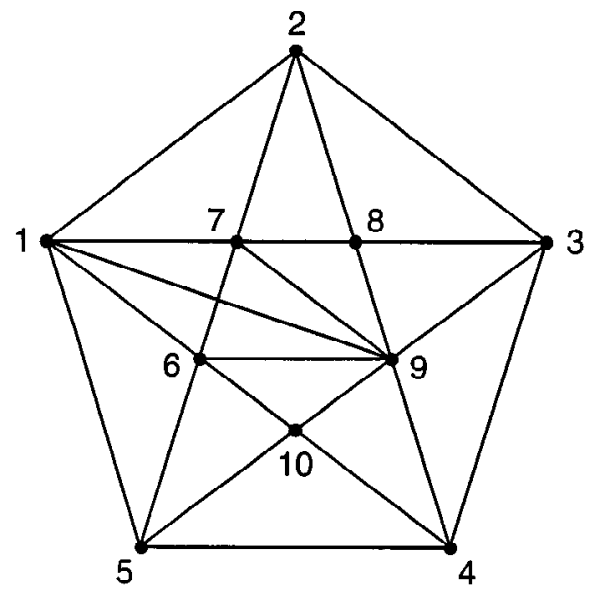

Fig. 3. Double $R_{5}: d(6,7)<d(6,9)=d(1,7)<d(1,2)=d(2,9)<d(1,9)<d(1,3)$.

latter option for all three $i$ gives $m=4$, so we assume $d(2,5)=d(2,1)=a$. If also $d(i, 5)=d(i, 1)$ for $i=3,4$, then 2,3 , and 4 are collinear, contradictory to 2,3 , and 4 on a circle centered at 1 . Thus, we can also assume $d(3,5)=d(1,5)=b$. It is easily seen that each possibility for $4, d(4,5)=d(4,1)=a$ and $d(4,5)=d(1,5)=b$, leads to a contradiction, so $m=3$ is impossible when $X$ is 3 -isosceles.

The discovery of larger 4-isosceles sets such as $C$ through $K$ on Fig. 1 has been aided by Theorem 2 and Fig. 3, where every noncollinear 4-set is isosceles. I do not believe that $R_{5}$ or $R_{5}^{+}$minus a vertex is included in any 4-isosceles set with more than eight points, but claim no proof. Our final result deals with $R_{4}$ and $R_{4}^{+}$.

Theorem 3. A 4-isosceles set that includes $R_{4}$ without its center, or that includes $R_{4}^{+}$, can have seven but not eight points.

Proof. Let $R_{4}=\{1,2,3,4\}$ with center $\circ$ as in Fig. 4. We note first that there are two convex 4-isosceles 7-sets that include $R_{4}$, namely $R_{4} \cup\{x, y, p\}$ and $R_{4} \cup\{x, y, q\}$ where $d(x, y)=d(x, 2)=d(x, p)$ and $d(3, q)=d(3,1)$. Neither admits an eighth point that preserves the 4-isosceles property.

We now consider other pairs of points that form 4-isosceles 6-sets with $R_{4}$, suppressing $\circ, 7,10$, and 13 initially. Such pairs include any like $\{17,18\}$ and $\{19,20\}$ with one point on the circle of radius $d(2,4)$ centered at 2 , the other on the circle of radius $d(2,4)$ centered at 4 , and line parallel to [2,4]. A similar remark holds for 1 and 3 in place of 2 and 4: see $\{11,12\}$. Other pairs like $\{15,16\}$ lie on a perpendicular bisector of a side of $R_{4}$ with points equidistant from the side. Another category, also infinite in number, allows one point but not the other to vary continuously. An example with fixed point 11 uses any sixth point on $\perp_{12}$ such as $6,8,9,15$, or 16. To avoid an isosceles 3 -set, the sixth point rules out 1 and 2 together and 3 and 4 together; 11 rules out the other four pairs from $R_{4}$. 


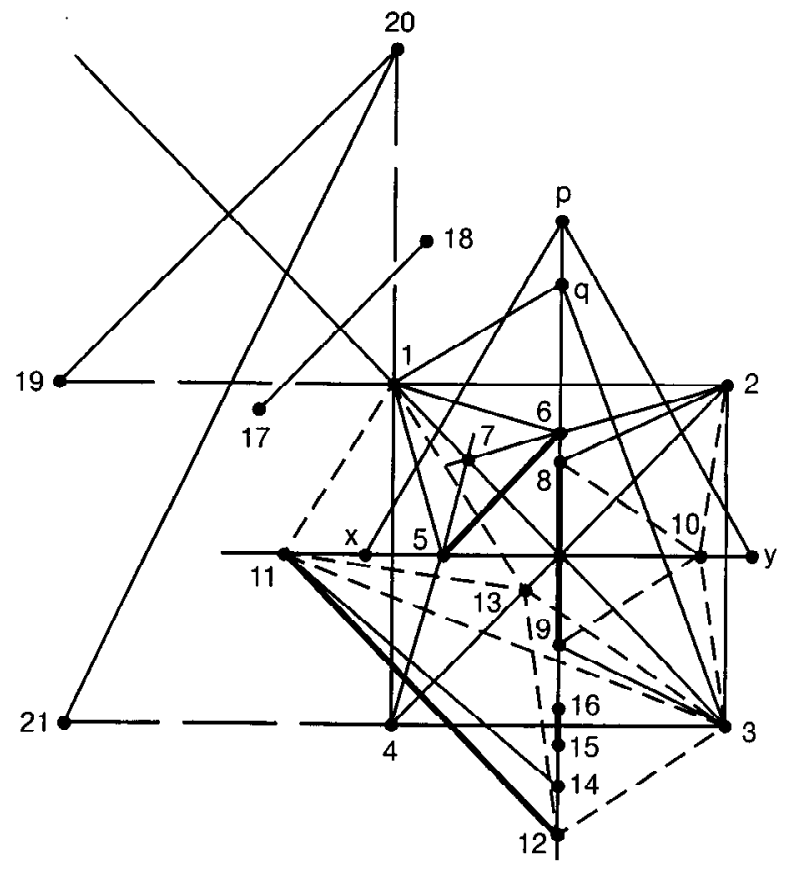

Fig. 4. $d(4,5)=d(5,6)=d(6,2), d(2,8)=d(8,9)=d(9,3), d(2,3)=d(3,6), d(4, \circ)=d(14, \circ)$.

A final set of pairs that exploit the geometry of the square is finite in number. The principals besides those in the first paragraph and those that use $\circ, 7,10$, and 13 are $\{20,21\}$ with $d(20,1)=d(1,2)=d(21,4), d(1,3)=d(1,21)$, and $d(20,21)=$ $d(2,21),\{11,14\}$ with $d(11,3)=d(1,3)$, and $d(14, \circ)=d(3, \circ),\{5,6\}$ with 5 and 6 on the circle of radius $d(3,2)$ centered at 3 and $d(2,6)=d(6,5)=d(5,4)$, and $\{8,9\}$ with $d(1,8)=d(2,8)=d(8,9)=d(9,3)$.

The points initially suppressed also lie in pairs that go with $R_{4}$ but serve the more interesting role of turning some 6-sets into 4-isosceles 7-sets. This happens with the center $\circ$ infinitely often, but the number of cases with seventh point elsewhere are finite. The three main types are illustrated by 7,10 , and 13 , with 7 at the center of the circle including $\{1,5,6\}, 10$ at the center of $\{2,3,8,9\}$, and 13 at the center of $\{1,3,11,12\}$. Each of $R_{4} \cup\{5,6,7\}, R_{4} \cup\{8,9,10\}$, and $R_{4} \cup\{11,12,13\}$ is 4-isosceles, and none remains 4-isosceles if $\circ$ or any other point is added. Addition of $\circ$ to $R_{4} \cup\{a, b\}$ gives a 4-isosceles 7-set when $\{a, b\}$ is a pair described in the preceding paragraphs on a circle centered at $\circ$, and in several other cases such as $\{11,14\}$, but none of these admits an eighth point that preserves the 4 -isosceles property.

\section{Open Questions}

Let $F$ denote a 4 -isosceles planar set.

Is there a 6-point $F$ with no 4 points on a circle and no 3 on a line? 
Is there a 7 -point $F$ with no 4 points on a circle?

Is there an 8-point $F$ whose points are the vertices of a convex octagon?

Describe all convex 7-point $F$.

Does Fig. 1 exhaust the 8-point $F$ sets?

Is there a 9 -point $F$ ?

\section{References}

1. E. Altman, On a problem of P. Erdös, Amer. Math. Monthly, 70 (1963), 148-157.

2. D. Avis, P. Erdös, and J. Pach, Distinct distances determined by subsets of a point set in space, Comput. Geom., 1 (1991), 1-11.

3. P. Erdös, Some combinatorial and metric problems in geometry, Colloquia Math. Soc. János Bolyai, 48 (1985), 167-177.

4. P. Erdös and P. Fishburn, Maximum planar sets that determine $k$ distances, Discrete Math., 160 (1996), $115-125$.

5. P. Erdös and P. Fishburn, Distinct distances in finite planar sets, Discrete Math., 175 (1997), 97-132.

6. P. Erdös and P. Fishburn, Duplicated distances in subsets of finite planar sets. Submitted.

7. P. Fishburn, Convex polygons with few intervertex distances, Comput. Geom., 5 (1995), 65-93.

Received February 1, 1997, and in revised form June 24, 1997. 